\title{
Line-Driven Ablation by External Irradiation
}

\author{
Kenneth G. Gayley, Stanley P. Owocki, and Steven R. Cranmer \\ 1 University of Iowa, Iowa City, IA, 52242, USA \\ ${ }^{2}$ Bartol Research Institute of the University of Delaware, Newark, DE 19716, USA \\ 3 Harvard-Smithsonian Center for Astrophysics, Cambridge, MA 02138, USA
}

\begin{abstract}
The Sobolev approximation for supersonic flows creates an effective opacity distribution that is nonisotropic, because the line-of-sight velocity gradient is different in different directions. To better understand the importance of this phenomenon in a simplified geometry, we consider line-driven flows in the planeparallel zero-sound-speed limit, and solve for the wind driven by radiation with an arbitrary angular distribution. One conclusion, surprising at first glance, is that the acceleration component normal to the surface is independent of both the strength and angular profile of the driving radiation field. The flow tilt and overall mass-loss rate do depend on the character of the radiation field. Also interesting is that mass loss through a surface may be generated or enhanced by irradiation that originates above the surface.
\end{abstract}

\section{Introduction}

The theory of radiatively driven mass loss, as grounded in the line-driving formalism developed by Castor, Abbott, and Klein (1975; hereafter CAK), has proven remarkably successful in explaining the general characteristics of stellar winds from hot, luminous, OB-type stars (e.g., Kudritzki \& Hummer 1990). In recent years there has developed considerable interest in applying this theory toward modeling radiatively driven mass flows in more complex geometries. Here we consider a plane-parallel atmosphere for simplicity, but include the effects of external irradiation superimposed against the intrinsic upwelling flux. In contrast with previous studies (Friend \& Castor 1983; Drechsel et al. 1995), we include the component of radiation reflected by the illuminated surface.

\section{Vertical Acceleration}

The momentum equation we apply normal to the surface is simply the standard CAK expression, and it leads directly to the surprising conclusion (see Gayley, Owocki, \& Cranmer 1998 for details) that the vertical acceleration is completely independent of the strength and angular distribution of the driving radiation field. This is a consequence of the assumption that the mass-loss rate stabilizes only once it achieves its maximum possible value, a generalization of the CAK "critical point" conditions. The overall magnitude of the 
driving intensity, as well as the total opacity, are then relevant only for fixing the mass-loss rate, while the angular character of the radiation also influences the azimuthal tilt of the flow.

\section{Azimuthal Force Equation}

To consider tilted flows, we must include the transverse from equation, which contains no gravity terms and involves different angular moments of the radiation field. Since the vertical acceleration is already known, we form the ratio of the transverse acceleration to the vertical, and after various constants in the radiative driving cancel, we are left with the essential angular moments. These moments depend on the angular anisotropy of the Sobolev opacity, so must be solved simultaneously with the wind dynamics to achieve self-consistency. The result is a nonlinear equation for the non-vertical flow direction, and its solution also determines the mass-loss rate.

\section{Results for an External Point Source}

The results for the simple example of irradiation from an external point source coupled with an intrinsic upwelling flux are shown in Figure (1a). An interesting conclusion from this figure is that the flow solution tends to align nearly perpendicular to the incident rays. Physically, this occurs because a wind that is perpendicular to the incident photon stream presents zero line-of-sight velocity gradient. Therefore, the downward photons slip easily through the wide frequency gaps between lines, whereas the upward reflected or intrinsic flux can couple strongly to the steep velocity gradient, supporting the mass-loss rate. This "bootstrap" quality is common in line-driven flows.

\section{Potential Effects of Finite Source Size and Disk Winds}

A perpendicular flow direction can only be defined for nearly parallel external irradiation, such as from a point source. If the external source is distributed, as for a nearby binary companion or the central source for a disk wind, the nearly perpendicular solution may cease to exist. Figure $1 \mathrm{~b}$ shows the parameter regimes for the various solutions for a spread external source. Notice the tendency for the solution to bifurcate to high tilt and low mass loss.

Although plane-parallel atmosphere models differ in fundamental ways from the physics of winds in other geometries, such as disk winds, our results may provide useful insight into the complexities induced by nonisotropic opacity in a broader context. For example, the blank region in Fig. (1b) indicates that no ablation occurs for strongly external illumination from a 


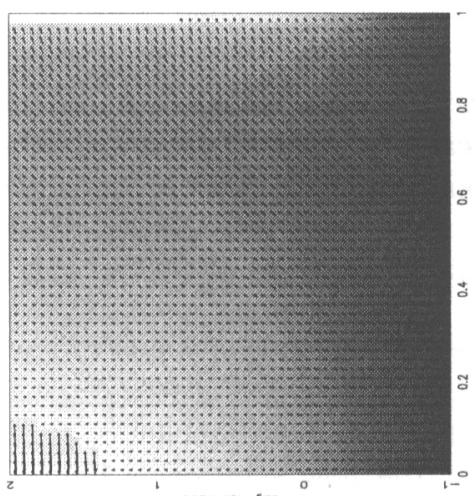

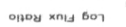

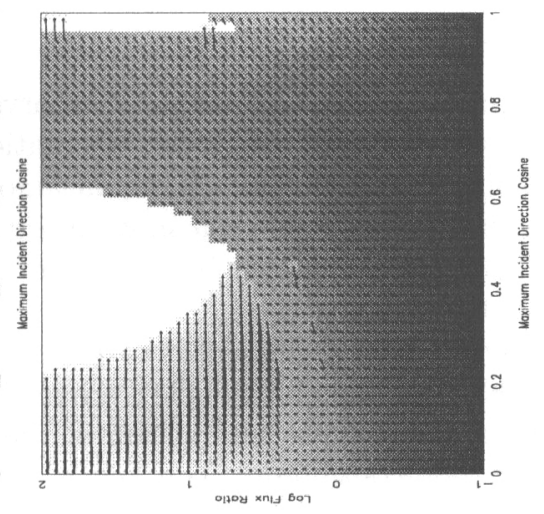

Fig. 1. a) Left: mass-loss-rate (gray scale) and flow direction (arrows) for outflow driven in part by irradiation from an external point source. The plot depicts a two-dimensional parameter space, with the external-to-intrinsic flux ratio at the bottom and the direction cosine to the external point source at the right. The flow direction is defined relative to an imaginary stellar surface at the right of the figure. b) Right: similar format to (a), except that the external source is spread from 0 to the maximum direction cosine indicated at the right.

distributed source with $\mu_{\max }>0.34$, since then there is simply too much downward pressure through which the wind cannot negotiate a path. This suggests it might prove difficult to use irradiation from a central source to drive a dynamically self-consistent wind from a disk inside about 3 times the source radius.

\section{Conclusions}

We stress that the two key elements that lead to surprising results in our models are the non-isotropic quality of the opacity, and the requirement for self-consistency between this opacity and the flow dynamics. Models which neglect either of these aspects may miss important characteristics of the ensuing flow.

This work was supported by NASA grants NAG5-4065, NAGW-2624, and NGT5-40024.

\section{References}

Castor, J. I., Abbott, D. C., \& Klein, R. I. 1975, ApJ, 195, 157 (CAK)

Gayley, K. G., Owocki, S. P., \& Cranmer, S. R. 1998, ApJ, in press

Drechsel, H., Haas, S., Lorenz, R., \& Gayler, S. 1995, A\&A, 294, 723

Friend, D. B., \& Castor, J. I. 1983, ApJ, 272, 259

Kudritzki, R.-P., \& Hummer, D. G. 1990, ARA\&A, 28, 303 


\section{Discussion}

A. Sapar: According to Sobolev theory, photons absorbed in a thin accelerating layer do not scatter monochromatically, but survive complete frequency redistribution and scatter in equal amounts to both sides of the scattering layer, thus giving to it their momentum before scattering. Backscattering effects can play a role only in the case of partial redistribution; but this is not the Sobolev scheme.

K. Gayley: The statement is correct, but the implication is not. Our models are purely within the Sobolev scheme and require no backscattering. All forces are due to the interaction of the non-isotropic Sobolev opacity with the incident continuum. The key is that the flow solution is dynamically self-consistent.

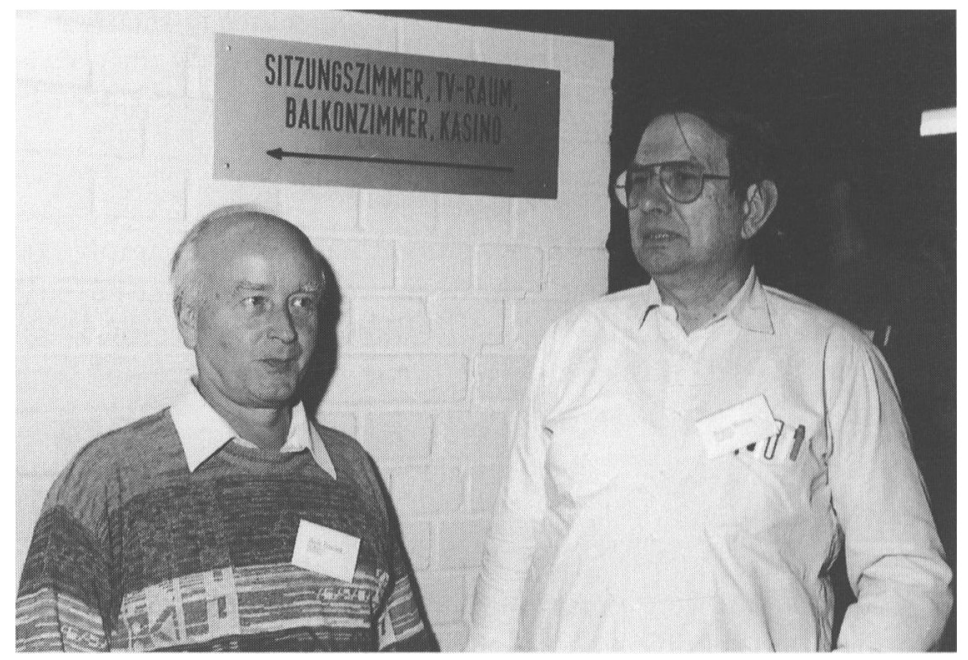

Bodo Baschek and Rainer Wehrse 